[Article]

\title{
正癸烷热解的小规模化学动力学机理模型
}

\author{
姚 通 钟北京* \\ (清华大学航天航空学院, 北京 100084)
}

\begin{abstract}
摘要: 正癸烷是目前常用的吸热型燃料的替代组分, 但是其热解机理的研究迄今还很少, 且现有的少数几个 机理由于规模庞大使用不便. 本文首先构建了一个包含 33 种组分和 75 个基元反应的正癸烷热解动力学机理模 型(Mech33); 随后, 在该机理的基础上进一步通过灵敏度分析得到影响主要热裂解组分生成的速率控制步, 并 采用局部平衡和稳态假设对 Mech33机理简化得到了规模更小的、仅包含 22 种组分和 59 步反应动力学机理模 型(Mech22). 在较宽的温度和压力范围内对流动反应器及激波管中正癸烷热解过程进行了数值模拟, 并与实 验数据进行了对比, 结果表明, Mech33 和 Mech22 两个动力学机理模型都能够很好地描述正癸烷热裂解过程, 并准确预测主要热裂解产物的浓度分布, 为进一步实现化学反应与计算流体力学(CFD)耦合的工程计算提供了 有价值的动力学机理模型.
\end{abstract}

关键词: 正癸烷; 热解; 化学动力学机理模型; 灵敏度分析; 简化模型

中图分类号: 0643

\section{Small-Scale Chemical Kinetic Mechanism Models for Pyrolysis of $n$-Decane}

\author{
YAO Tong ZHONG Bei-Jing* \\ (School of Aerospace, Tsinghua University, Beijing 100084, P. R. China)
}

\begin{abstract}
Decane is a component of commonly used fuels, but so far studies into its pyrolysis mechanism are rare and the few existing mechanisms are inconvenient to use owing to their large scales. A small-scale chemical kinetic model (Mech33) for describing the process of $n$-decane pyrolysis containing 33 species and 75 elementary reactions was constructed. Based on partial equilibrium and quasi-steady state assumptions through sensitivity analysis, a smaller kinetic model (Mech22) containing 22 species and 59 reactions was developed from Mech33. Simulations of $n$-decane pyrolysis using these two models were compared with experimental data from flow reactor and shock tube over a wide range of temperatures and pressures. The results showed that Mech33 and Mech22 could reproduce the process of $n$-decane pyrolysis well and accurately predict the concentrations profile of main products, and finally provide valuable chemical kinetic models for engineering simulations when coupled with computational fluid dynamics (CFD).
\end{abstract}

Key Words: $n$-Decane; Pyrolysis; Chemical kinetic mechanism model; Sensitivity analysis; Reduced model

\section{1 引言}

超燃冲压发动机是高速导弹、高超声速飞行器
以及航天器实现高超声速飞行的关键, 其设计研制 非常复杂, 其中一个关键问题是发动机壁面冷却和

Received: February 26, 2013; Revised: April 12, 2013; Published on Web: April 12, 2013.

"Corresponding author. Email: zhongbj@tsinghua.edu.cn; Tel: +86-10-62772928.

The project was supported by the National Natural Science Foundation of China (51036004).

国家自然科学基金(51036004)资助项目

C. Editorial office of Acta Physico-Chimica Sinica 
热管理问题.'目前提出了一种再生冷却方式, 即将 吸热型碳氢燃料作为冷却剂来冷却发动机壁面, 利 用燃料的热沉(物理热沉和化学热沉), 吸收发动机 燃烧室放出的热量, 在不增加飞行器负担情况下实 现了对发动机的有效冷却. ${ }^{2-4}$ 同时, 燃料吸热发生了 裂解反应, 生成易于着火和燃烧的小分子进入燃烧 室进行燃烧, 有助于改善燃烧过程, 提高能量的利 用率. 为了研究燃料裂解热沉和裂解吸热的效果, 需要对航空燃料裂解动力学进行深入的研究.

但是, 航空燃料是由包括链烷烃、环烷烃、芳香 烃在内的上百种化合物组成的复杂混合物, 对其直 接进行详细机理的研究目前还存在许多困难. 由于 正癸烷是航空煤油重要组分, 常用作单组分替代燃 料 ${ }^{5}$ 或多组分替代燃料的一种组分 ${ }^{6,7}$ 来研究煤油特 性, 因此对于正癸烷热解动力学的研究具有重要的 意义. 然而, 目前对于正癸烷热裂解机理的研究还 非常少, Yu 和 Eser ${ }^{8}$ 研究了温度 673-723 K、压力 1.26-7.24 MPa 临界和超临界状态下包括正癸烷在 内的 $\mathrm{C}_{10}-\mathrm{C}_{14}$ 链烷烃的热解动力学, 得到了链烷烃与 碳原子个数相关联的热裂解一阶反应动力学常数, 但无法描述热解详细动力学过程; 焦毅等 ${ }^{9}$ 通过自 制常压裂解装置, 研究了正癸烷在温度范围为 973-1123 K、停留时间为 0.5-2 s 的裂解产物随停留 时间的变化, 并通过机理生成程序得到了 281 种组 分和 1072 个反应的正癸烷裂解机理, 但是这个机理 不仅不能准确地预报其自身的实验结果, 而且还存 在较大的偏差. 此外, 由于该机理规模过于庞大, 不 适用于多维湍流数值模拟; Zeppieri 等 ${ }^{10}$ 在正庚烷机 理基础上发展了一个正癸烷裂解和氧化反应机理 (69 种组分和 494 个反应)机理, 并通过简化得到了 52 种组分和 407 个反应骨架机理. 但是, 与实验结果 的对比表明, 该机理模型的预报结果与实验结果吻 合程度较差. ${ }^{11}$ Lawrence 实验室的 Westbrook 等 ${ }^{12}$ 发 展出了适用于 $\mathrm{C}_{8}-\mathrm{C}_{16}$ 的直链烷烃氧化和裂解的动力 学模型, 该模型包含了 2116 种组分和 8130 个反应, 对正癸烷在流动反应器中的裂解过程进行了模拟, 计算结果与实验结果符合情况良好, 但是该机理规 模过于庞大, 很难用于与多维计算流体力学 (CFD) 的耦合计算. 因此, 能够实现准确预报正癸烷热解 过程的化学反应机理的研究仍然需要进一步完善. 本文的目的是在现有的正癸烷热解机理的基础上 构建一个能够比较准确描述正癸烷热解过程, 并预 测主要热解产物分布的、规模较小的正癸烷热解化
学反应机理模型, 在提高对正癸烷裂解过程及主要 产物分布预测准确性的同时, 为实现与多维计算流 体力学耦合计算的工程应用提供有价值的动力学 模型.

\section{2 正癸烷裂解动力学机理模型构建}

许多研究 ${ }^{10,13-16}$ 表明高碳氢燃料热裂解主要包 括以下几个动力学过程: 燃料分子裂解和脱氢反 应、烷基的 $\beta$ 断键反应、烯烃的脱氢和裂解反应、以 及小分子及其自由基的反应. 根据 Zeppieri 等 ${ }^{10}$ 和 Malewicki和 Brezinsky ${ }^{17}$ 的实验研究, 正癸烷裂解的 主要产物包括 $\mathrm{C}_{2} \mathrm{H}_{4} 、 \mathrm{CH}_{4} 、 \mathrm{C}_{3} \mathrm{H}_{6} 、 \mathrm{C}_{4} \mathrm{H}_{8} 、 \mathrm{C}_{2} \mathrm{H}_{6}$ 和 $\mathrm{C}_{2} \mathrm{H}_{2}$, 且 不同条件下这些产物中碳含量的总和占到了正癸 烷总碳量的 78.9\%-96.1\%. 因此, 要准确预测正癸烷 热裂解过程, 以及热裂解产物的分布和热沉, 除了 构建大碳氢分子分解的化学反应动力学机理外, 同 时还必须有准确描述小分子烷烃和烯烃分子的动 力学机理. 小分子碳氢的动力学机理有许多学者进 行过详细的研究和讨论, ${ }^{18-20}$ 本文有关 $\mathrm{C}_{0}-\mathrm{C}_{3}$ 小分子 及其自由基的反应直接通过反应动力学分析引自 文献 ${ }^{21}$ 裂解机理的核心部分, 因此下面主要讨论 $\mathrm{C}_{4}$ 及以上分子的热裂解动力学机理.

\section{1 燃料分子裂解和脱氢反应}

正癸烷的消耗主要通过正癸烷单分子裂解反 应和脱氢反应进行. 正癸烷热解的起始反应步是 $\mathrm{C}-\mathrm{C}$ 键或 $\mathrm{C}-\mathrm{H}$ 键的断裂, 生成两个烷基或者癸烷 基和氢原子的反应. 但是, 由于 $\mathrm{C}-\mathrm{H}$ 键能较高, 相 比而言, $\mathrm{C}-\mathrm{C}$ 键的断裂较 $\mathrm{C}-\mathrm{H}$ 键的断裂更易于发 生, 因此本文主要考虑正癸烷的 $\mathrm{C}-\mathrm{C}$ 键断裂反应. 根据 Peters 等 ${ }^{21,22}$ 和 Olchanski 和 Burcat ${ }^{23}$ 的研究, 正 癸烷单分子裂解反应产物包括 $\mathrm{C}_{1}-\mathrm{C}_{9}$ 的烷基. 为了 减少热裂解机理涉及的组分数和反应数, 根据对机 理进行的反应流分析结果, ${ }^{21-23}$ 本文的动力学机理模 型只考虑下面的两个主要裂解反应路径:

$$
\begin{aligned}
& n-\mathrm{C}_{10} \mathrm{H}_{22} \rightarrow p-\mathrm{C}_{4} \mathrm{H}_{9}+1-\mathrm{C}_{6} \mathrm{H}_{13} \\
& n-\mathrm{C}_{10} \mathrm{H}_{22} \rightarrow n-\mathrm{C}_{3} \mathrm{H}_{7}+1-\mathrm{C}_{7} \mathrm{H}_{15}
\end{aligned}
$$

并通过修正反应动力学参数来弥补删除路径造成 的误差. 由于 $\mathrm{C}-\mathrm{C}$ 键断裂是单分子生成双分子的 反应, 会带来压力降低引起反应速率常数降低的 fall-off效应, 目前, 尚无实验或理论计算研究过 $\mathrm{C}_{5}$ 以上烷烃的 $\mathrm{C}-\mathrm{C}$ 解离速率随温度和压力的变化. 现有较权威的大分子碳氢燃料氧化及裂解机理中, 研究者对于 $\mathrm{C}_{5}$ 以上烷烃 $\mathrm{C}-\mathrm{C}$ 键裂解的 fall-off 效应 
都不予考虑. 因此, 这里我们只考虑 $\mathrm{C}_{4}$ 以下烷烃裂 解反应中的 fall-off 效应, 不考虑正癸烷直接裂解 反应中压力 fall-off 效应. 许多研究者对上述正癸 烷 $\mathrm{C}-\mathrm{C}$ 键断裂反应 $\mathrm{R} 1$ 和 $\mathrm{R} 2$ 给出了相同的动力学 参数. Peters 等 ${ }^{21,22}$ 在其正癸烷燃烧机理中给出的指 前因子为 $3.16 \times 10^{16} \mathrm{~s}^{-1}$, 活化能为 $399-343 \mathrm{~kJ} \cdot \mathrm{mol}^{-1}$; Olchanski 和 Burcat ${ }^{23}$ 在 Peters 机理参数的基础上乘 以 1.5-2.5 的系数, 得到的指前因子为 $4.3 \times 10^{16}-6.5 \times$ $10^{16} \mathrm{~s}^{-1}$, 而活化能采用和 Peters 一样的数值; Ranzi 等 ${ }^{24}$ 在其发展的正癸烷热解及氧化反应模型中给出 的指前因子为 $1 \times 10^{17} \mathrm{~s}^{-1}$, 活化能为 $399 \mathrm{~kJ} \cdot \mathrm{mol}^{-1}$; Zeppieri 等 ${ }^{10}$ 在其发展的正癸烷裂解模型中给出的 指前因子为 $2 \times 10^{17} \mathrm{~s}^{-1}$, 活化能为 $348-352 \mathrm{~kJ} \cdot \mathrm{mol}^{-1}$; Battin-Leclerc 模型 25 给出的活化能为 348-364 kJ $\mathrm{mol}^{-1}$; Lindstedt 和 Maurice ${ }^{26}$ 在其燃烧反应模型中给 出的指前因子为 $0.785 \times 10^{17}-1.047 \times 10^{17} \mathrm{~s}^{-1}$, 而活化 能采用了较小的值, 为 $285 \mathrm{~kJ} \cdot \mathrm{mol}^{-1}$. 可见, 反应 $\mathrm{R} 1$ 和 $\mathrm{R} 2$ 的动力学参数存在较大的分散性. 为了使正癸 烷热裂解产物计算值与实验结果吻合更好, 本文经 过一系列的动力学参数的调整, 最后采用的这两个 反应的指前因子为 $2.62 \times 10^{17} \mathrm{~s}^{-1}$, 而活化能采用和 Zeppieri机理中一样的数值.

正癸烷消耗的另外一种反应类型是有自由基 参与的正癸烷脱氢反应. 自由基攻击正癸烷分子, 使其失去一个 $\mathrm{H}$ 原子从而产生正癸烷基. Westbrook 等 ${ }^{12}$ 的研究表明, 根据脱氢碳位的不同, 正癸烷脱氢 反应可以生成五种正癸烷基的同分异构体. 为了减 小机理规模, 可以采用具有代表性的同分异构体来 代替. 但是, 也有研究表明, 完全不考虑烷基同分异 构体之间的异构化反应, 容易造成动力学机理模型 高估乙烯产率的计算结果, ${ }^{27}$ 因此, 在 Olchanski 机 理 ${ }^{23}$ 中考虑了三种同分异构体, 而在 Peters 机理 ${ }^{21,22}$ 中考虑了两种同分异构体. 本文根据 Peters 等 ${ }^{21,22}$ 的 建议, 考虑 2- $\mathrm{C}_{10} \mathrm{H}_{21}$ 和 3- $\mathrm{C}_{10} \mathrm{H}_{21}$ 两种比较重要的同分 异构体, 以及这两种同分异构体之间的氢迁移重排 反应, 以保证对乙烯产率预测的准确性. 在热解过 程中, 正癸烷分子脱氢主要是通过与自由基 $\mathrm{H}$ 和 $\mathrm{CH}_{3}$ 的反应, 而与其他自由基的反应较少, ${ }^{28}$ 因此在 本文的模型中, 我们只考虑 $\mathrm{H}$ 和 $\mathrm{CH}_{3}$ 自由基与正癸 烷分子的反应.

$$
\begin{aligned}
& n-\mathrm{C}_{10} \mathrm{H}_{22}+\mathrm{CH}_{3} \rightarrow 3-\mathrm{C}_{10} \mathrm{H}_{21}+\mathrm{CH}_{4} \\
& n-\mathrm{C}_{10} \mathrm{H}_{22}+\mathrm{CH}_{3} \rightarrow 2-\mathrm{C}_{10} \mathrm{H}_{21}+\mathrm{CH}_{4} \\
& n-\mathrm{C}_{10} \mathrm{H}_{22}+\mathrm{H} \rightarrow 3-\mathrm{C}_{10} \mathrm{H}_{21}+\mathrm{H}_{2}
\end{aligned}
$$

$$
\begin{aligned}
& n-\mathrm{C}_{10} \mathrm{H}_{22}+\mathrm{H} \rightarrow 2-\mathrm{C}_{10} \mathrm{H}_{21}+\mathrm{H}_{2} \\
& 2-\mathrm{C}_{10} \mathrm{H}_{21} \rightarrow 3-\mathrm{C}_{10} \mathrm{H}_{21} \\
& 3-\mathrm{C}_{10} \mathrm{H}_{21} \rightarrow 2-\mathrm{C}_{10} \mathrm{H}_{21}
\end{aligned}
$$

对于正癸烷脱氢反应速率常数, 根据 Tamura ${ }^{29}$ 的研究结果, 脱氢反应的指前因子与脱氢碳位上 的氢原子个数有关. 由于 $2-\mathrm{C}_{10} \mathrm{H}_{21}$ 和 $3-\mathrm{C}_{10} \mathrm{H}_{21}$ 脱氢碳 位上的氢原子个数相等, 因此, 反应 $\mathrm{R} 3$ 与 $\mathrm{R} 4$ 以及 R5 与 R6 的指前因子和活化能相等. Olchanski 和 Burcat $^{23}$ 在正庚烷氧化机理 ${ }^{28}$ 基础上, 推断出了正癸 烷脱氢反应指数前因子, 正癸烷与 $\mathrm{CH}_{3}$ 的反应生成 $2-\mathrm{C}_{10} \mathrm{H}_{21}$ 和 3- $\mathrm{C}_{10} \mathrm{H}_{21}$ 的指前因子为 $3.2 \times 10^{12} \mathrm{~mol}^{-1}$. $\mathrm{cm}^{3} \cdot \mathrm{s}^{-1}$, 与 $\mathrm{H}$ 反应的指前因子为 $1.91 \times 10^{7} \mathrm{~mol}^{-1} \cdot \mathrm{cm}^{3}$. $\mathrm{s}^{-1}$; Peters 等 ${ }^{21,22}$ 在他们的正癸烷氧化动力学机理模 型中给出了正癸烷与自由基 $\mathrm{CH}_{3}$ 和 $\mathrm{H}$ 反应的指前因 子分别为 $1.0 \times 10^{12}$ 和 $4.5 \times 10^{7} \mathrm{~mol}^{-1} \cdot \mathrm{cm}^{3} \cdot \mathrm{s}^{-1}$. 由于热 解模型中消耗正癸烷的自由基只考虑了 $\mathrm{H}$ 和 $\mathrm{CH}_{3}$, 而没有氧化模型中正癸烷与 $\mathrm{OH} 、 \mathrm{H}_{2} \mathrm{O}_{2}$ 等含氧自由 基的反应, 因此本文适当调整了正癸烷与 $\mathrm{CH}_{3}$ 和与 $\mathrm{H}$ 反应的指前因子分别为 $2.35 \times 10^{13}$ 和 $1.05 \times 10^{9}$ $\mathrm{mol}^{-1} \cdot \mathrm{cm}^{3} \cdot \mathrm{s}^{-1}$. 对于氢迁移重排反应的动力学参数 仍采用Peters 等 ${ }^{21,22}$ 建议的参数.

\section{2 烷基的 $\beta$ 断键反应}

烷烃的热解过程中生成的烷基自由基的裂解 对中间组分的分布和系统反应性有重要影响. 癸烷 基主要通过 $\beta$ 断键法则断裂, 生成更小的烷基和烯 烃. 这里我们只考虑如下几个主要的反应路径:

$$
\begin{aligned}
& 3-\mathrm{C}_{10} \mathrm{H}_{21} \rightarrow 1-\mathrm{C}_{8} \mathrm{H}_{17}+\mathrm{C}_{2} \mathrm{H}_{4} \\
& 2-\mathrm{C}_{10} \mathrm{H}_{21} \rightarrow 1-\mathrm{C}_{7} \mathrm{H}_{15}+\mathrm{C}_{3} \mathrm{H}_{6} \\
& 3-\mathrm{C}_{10} \mathrm{H}_{21} \rightarrow 1-\mathrm{C}_{6} \mathrm{H}_{13}+1-\mathrm{C}_{4} \mathrm{H}_{8} \\
& 2-\mathrm{C}_{10} \mathrm{H}_{21} \rightarrow 1-\mathrm{C}_{5} \mathrm{H}_{11}+1-\mathrm{C}_{5} \mathrm{H}_{10}
\end{aligned}
$$

Peters 等 ${ }^{21,22}$ 给出了反应 $\mathrm{R} 10$ 和反应 $\mathrm{R} 11$ 的指前 因子和活化能分别为 $2.5 \times 10^{13} \mathrm{~s}^{-1}$ 和 $116.4 \mathrm{~kJ} \cdot \mathrm{mol}^{-1}$; Olchanski和 Burcat ${ }^{23}$ 给出的指前因子和活化能分别 为 $1.0 \times 10^{13} \mathrm{~s}^{-1}$ 和 $118.5 \mathrm{~kJ} \cdot \mathrm{mol}^{-1}$. 本文采用 $\mathrm{R} 10$ 和 $\mathrm{R} 11$ 的动力学参数分别是指前因子为 $1.5 \times 10^{13} \mathrm{~s}^{-1}$, 活 化能为 $118.5 \mathrm{~kJ} \cdot \mathrm{mol}^{-1}$; 而反应 $\mathrm{R} 9$ 和 $\mathrm{R} 12$ 采用了与 $\mathrm{R} 10$ 和 $\mathrm{R} 11$ 相同的参数.

同样, 辛烷基和庚烷基主要通过 $\beta$ 断键反应生 成烷基和烯烃:

$$
\begin{aligned}
& 1-\mathrm{C}_{8} \mathrm{H}_{17} \rightleftharpoons 1-\mathrm{C}_{7} \mathrm{H}_{14}+\mathrm{CH}_{3} \\
& 1-\mathrm{C}_{8} \mathrm{H}_{17} \rightleftharpoons 1-\mathrm{C}_{6} \mathrm{H}_{13}+\mathrm{C}_{2} \mathrm{H}_{4} \\
& 1-\mathrm{C}_{8} \mathrm{H}_{17} \rightleftharpoons 1-\mathrm{C}_{5} \mathrm{H}_{11}+\mathrm{C}_{3} \mathrm{H}_{6} \\
& 1-\mathrm{C}_{8} \mathrm{H}_{17} \rightleftharpoons p-\mathrm{C}_{4} \mathrm{H}_{9}+1-\mathrm{C}_{4} \mathrm{H}_{8}
\end{aligned}
$$


$1-\mathrm{C}_{7} \mathrm{H}_{15} \rightarrow 1-\mathrm{C}_{6} \mathrm{H}_{12}+\mathrm{CH}_{3}$

$1-\mathrm{C}_{7} \mathrm{H}_{15} \rightarrow 1-\mathrm{C}_{5} \mathrm{H}_{10}+\mathrm{C}_{2} \mathrm{H}_{5}$

$1-\mathrm{C}_{7} \mathrm{H}_{15} \rightarrow p-\mathrm{C}_{4} \mathrm{H}_{9}+\mathrm{C}_{3} \mathrm{H}_{6}$

$1-\mathrm{C}_{7} \mathrm{H}_{15} \rightarrow 1-\mathrm{C}_{4} \mathrm{H}_{8}+n-\mathrm{C}_{3} \mathrm{H}_{7}$

上述辛烷基的断键反应 R13-R16 与 Chakir 等 ${ }^{28}$ 给出的庚烷基断键反应类似, 因此我们采用与庚烷 基裂解反应相同的动力学参数; 而反应 R17-R20 的 动力学参数直接采用 Peters 等 ${ }^{21,22}$ 给出的建议值.

\section{3 烯烃的脱氢和裂解反应}

烯烃的消耗主要通过脱氢反应生成相应的更 小烷基, 并进一步分解成小分子烷基和烯烃, 为了 减少组分数和反应数, 本文采用 Peters 等 ${ }^{21,22}$ 的总包 表达方式来模拟此类反应的动力学反应过程 (R21-R24、R27-R30、R32-R34、R40-R42). 但是直 接采用 Peters 模型中的参数进行计算, 结果对 $\mathrm{C}_{3} \mathrm{H}_{6}$ 和 $\mathrm{C}_{2} \mathrm{H}_{2}$ 预测偏低. 灵敏度分析结果表明, 反应 $1-\mathrm{C}_{7} \mathrm{H}_{14}+\mathrm{C}_{2} \mathrm{H}_{5} \rightarrow \mathrm{C}_{2} \mathrm{H}_{6}+\mathrm{C}_{2} \mathrm{H}_{4}+\mathrm{C}_{4} \mathrm{H}_{6}+\mathrm{CH}_{3}$ (R24) 对于中 间组分 $\mathrm{C}_{5} \mathrm{H}_{10}$ 的浓度影响较大, 由于反应 $\mathrm{R} 24$ 并非基 元反应形式, 而是采用总包反应形式, Peters 等的模 型中此反应对于 $\mathrm{C}_{5} \mathrm{H}_{10}$ 的预测偏高, 表明该反应速率 偏低, 为了使模型对于小分子产物 $\mathrm{C}_{3} \mathrm{H}_{6}$ 和 $\mathrm{C}_{2} \mathrm{H}_{2}$ 的预 测与实验结果达到最大程度的吻合, 需要提高该反 应的速率, 根据文献 ${ }^{30}$ 中动力学参数调整的原则, 提 高反应R24指前因子, 经过尝试, 最终将该反应指前 因子提高 10 倍, 并增加反应 1- $\mathrm{C}_{5} \mathrm{H}_{10} \rightarrow \mathrm{C}_{3} \mathrm{H}_{6}+\mathrm{C}_{2} \mathrm{H}_{2}+\mathrm{H}_{2}$ (R35), 其动力学参数采用与反应 $\mathrm{R} 32$ 相同的活化 能, 指前因子进行微调, 以增加 $\mathrm{C}_{5} \mathrm{H}_{10}$ 的转化, 提高对 于 $\mathrm{C}_{3} \mathrm{H}_{6}$ 和 $\mathrm{C}_{2} \mathrm{H}_{2}$ 预测的准确性. 由于在 Peters 等 ${ }^{21,22}$ 的 模型中缺少 $\mathrm{C}_{4} \mathrm{H}_{8}$ 的反应路径, 从而导致 $\mathrm{C}_{4} \mathrm{H}_{8}$ 的预测 出现偏差. 考虑到 $\mathrm{C}_{4} \mathrm{H}_{8}$ 的消耗主要通过脱氢反应进 行, 因此, 我们增加 $1-\mathrm{C}_{4} \mathrm{H}_{8}+\mathrm{H} \rightleftharpoons \mathrm{H}_{2}+\mathrm{C}_{4} \mathrm{H}_{6}+\mathrm{H}(\mathrm{R} 42)$ 反 应, ${ }^{31}$ 其动力学参数参照了张云鹏等 ${ }^{32}$ 提出的正庚烷 模型的参数.

我们采用上述方法得到的正癸烷热裂解主要 反应构成了 $\mathrm{C}_{4}$ 及以上组分的动力学机理模型, 加上 我们基于文献 ${ }^{21,22}$ 得到的 $\mathrm{C}_{0}-\mathrm{C}_{3}$ 小分子反应, 就构建 了本文包含 33 种组分和 75 个基元反应的正癸烷热 裂解反应动力学机理模型(Mech33), 具体的反应及 其动力学参数如表 1 所示. 所有物种的热力学数据 来自文献. ${ }^{21,22}$

\section{3 正癸烷裂解动力学机理模型的验证}

上述构建的正癸烷热裂解反应动力学机理模
型(Mech33)包括两个子模型: (1) $\mathrm{C}_{0}-\mathrm{C}_{3}$ 模型, 包含 $\mathrm{C}_{0}-\mathrm{C}_{3}$ 小分子及其自由基的反应, 是热裂解机理的 核心部分, 对于小分子产物的产率分布具有较大影 响; (2) $\mathrm{C}_{4}$ 及以上模型, 描述了 $\mathrm{C}_{4}$ 以上的分子热裂解 反应的动力学机理模型, 包括起始步燃料分子 $\mathrm{C}-$ $\mathrm{C}$ 键的断裂反应、燃料分子脱氢反应、烷基的氢迁移 重排反应、烷基的 $\beta$-键断裂分解反应、烯烃的脱氢反 应以及烯烃的分解反应. 目前对于正癸烷热解的实 验研究较少, 我们利用现有的实验数据, 采用流动 反应器和激波管中的裂解过程来对动力学机理模 型进行验证.

\section{1 流动反应器}

我们通过比较 Mech33 的计算结果与 Zeppieri 等 ${ }^{10}$ 的实验数据来验证上述热裂解反应机理的合理 性. 数值计算采用 CHEMKIN 中的管流模型, ${ }^{33}$ 计算 条件如下: 热裂解温度 $T=1060 \mathrm{~K}$, 压力 $p=0.1 \mathrm{MPa}$, 正癸烷在稀释气体 $\mathrm{N}_{2}$ 中的摩尔分数为 $1.456 \times 10^{-3}$. 计算得到了上述条件下正癸烷及其主要热裂解产 物的摩尔分数随反应停留时间的分布. Zeppieri 认 为, 由于实验设备和测量仪器的误差, 初始的时间 测点与实际情况有偏差, 因而组分浓度测量是不准 确的, 因此, Zeppieri 建议实验数据点整体向后移动 (即相应的时间点右移) $33 \mathrm{~ms}$, 这样保证了Zeppieri 的实验结果和他们自己的计算值相吻合. ${ }^{10}$

图 1 给出了实验测量 ${ }^{10}$ 和动力学机理模型预测 的正癸烷 $\left(n-\mathrm{C}_{10} \mathrm{H}_{22}\right)$ 及其主要的热裂解产物 $\left(\mathrm{CH}_{4}\right.$ 、 $\mathrm{C}_{2} \mathrm{H}_{2} 、 \mathrm{C}_{2} \mathrm{H}_{4} 、 \mathrm{C}_{2} \mathrm{H}_{6} 、 \mathrm{C}_{3} \mathrm{H}_{6}$ 和 $\mathrm{C}_{4} \mathrm{H}_{8}$ ) 的摩尔分数随反应时 间的变化. 图 1 同时给出了使用本文的热裂解机理、 Lawrence 机理 ${ }^{12}$ 和 Zeppieri 机理 ${ }^{10}$ 的计算结果. 从图 1(a)中可以看出, Zeppieri机理对于正癸烷转化速率 的预测偏低, 在 $0.3 \mathrm{~s}$ 的停留时间内, 预测的正癸烷 摩尔分数始终高于实验结果, Lawrence 机理 ${ }^{12}$ 和本 文构建的 Mech33 机理则能够很好地预测整个热解 过程中正癸烷的转化; 此外 Zeppieri 机理 ${ }^{10}$ 对于 $\mathrm{C}_{2} \mathrm{H}_{4}$ 的预测偏低, 而 Lawrence 机理 ${ }^{2}$ 则明显高估了 $\mathrm{C}_{2} \mathrm{H}_{4}$ 的产量. 图 1(b)中, Zeppieri 机理 ${ }^{10}$ 对于 $\mathrm{C}_{3} \mathrm{H}_{6}$ 的生成 量的预测高出两倍, 说明 Zeppieri机理 ${ }^{10}$ 中 $\mathrm{C}_{3}$ 水平上 的子机理需要进一步的优化和修正, Lawrence 机 理 ${ }^{12}$ 和 Mech33 机理对于 $\mathrm{C}_{3} \mathrm{H}_{6}$ 的计算结果与实验值 符合较好. 图 1(c)中, Zeppieri机理对于 $\mathrm{CH}_{4}$ 最终生 成量的预测偏高. 图 1(d)和(e)中, Lawrence机理对 $\mathrm{C}_{2} \mathrm{H}_{6}$ 的预测偏低, 而对于 $\mathrm{C}_{2} \mathrm{H}_{2}$ 的计算结果则偏差更 大. 图 1(f)中, Lawrence 机理和 Zeppieri 机理对于 
表 1 正癸烷热裂解反应动力学机理模型参数(Mech33)

Table 1 Rate parameters of chemical kinetic mechanism for pyrolysis of $\boldsymbol{n}$-decane (Mech 33)

\begin{tabular}{|c|c|c|c|c|c|}
\hline No. & Reaction & $A$ & $n$ & $E /\left(\mathrm{kJ} \cdot \mathrm{mol}^{-1}\right)$ & Ref. \\
\hline 1 & $n-\mathrm{C}_{10} \mathrm{H}_{22} \rightarrow p-\mathrm{C}_{4} \mathrm{H}_{9}+1-\mathrm{C}_{6} \mathrm{H}_{13}$ & $2.62 \times 10^{17}$ & 0 & 348.26 & this work \\
\hline 2 & $n-\mathrm{C}_{10} \mathrm{H}_{22} \rightarrow n-\mathrm{C}_{3} \mathrm{H}_{7}+1-\mathrm{C}_{7} \mathrm{H}_{15}$ & $2.62 \times 10^{17}$ & 0 & 349.18 & this work \\
\hline 3 & $n-\mathrm{C}_{10} \mathrm{H}_{22}+\mathrm{CH}_{3} \rightarrow 3-\mathrm{C}_{10} \mathrm{H}_{21}+\mathrm{CH}_{4}$ & $2.35 \times 10^{13}$ & 0 & 35.97 & this work \\
\hline 4 & $n-\mathrm{C}_{10} \mathrm{H}_{22}+\mathrm{CH}_{3} \rightarrow 2-\mathrm{C}_{10} \mathrm{H}_{21}+\mathrm{CH}_{4}$ & $2.35 \times 10^{13}$ & 0 & 35.97 & this work \\
\hline 5 & $n-\mathrm{C}_{10} \mathrm{H}_{22}+\mathrm{H} \rightarrow 3-\mathrm{C}_{10} \mathrm{H}_{21}+\mathrm{H}_{2}$ & $1.05 \times 10^{9}$ & 2.0 & 20.91 & this work \\
\hline 6 & $n-\mathrm{C}_{10} \mathrm{H}_{22}+\mathrm{H} \rightarrow 2-\mathrm{C}_{10} \mathrm{H}_{21}+\mathrm{H}_{2}$ & $1.05 \times 10^{9}$ & 2.0 & 20.91 & this work \\
\hline 7 & $2-\mathrm{C}_{10} \mathrm{H}_{21} \rightarrow 3-\mathrm{C}_{10} \mathrm{H}_{21}$ & $2.00 \times 10^{9}$ & 0 & 75.85 & 21,22 \\
\hline 8 & $3-\mathrm{C}_{10} \mathrm{H}_{21} \rightarrow 2-\mathrm{C}_{10} \mathrm{H}_{21}$ & $2.00 \times 10^{9}$ & 0 & 75.85 & 21,22 \\
\hline 9 & 3- $\mathrm{C}_{10} \mathrm{H}_{21} \rightarrow 1-\mathrm{C}_{8} \mathrm{H}_{17}+\mathrm{C}_{2} \mathrm{H}_{4}$ & $1.50 \times 10^{13}$ & 0 & 118.38 & 21,22 \\
\hline 10 & $2-\mathrm{C}_{10} \mathrm{H}_{21} \rightarrow 1-\mathrm{C}_{7} \mathrm{H}_{15}+\mathrm{C}_{3} \mathrm{H}_{6}$ & $1.50 \times 10^{13}$ & 0 & 118.38 & 21,22 \\
\hline 11 & $3-\mathrm{C}_{10} \mathrm{H}_{21} \rightarrow 1-\mathrm{C}_{6} \mathrm{H}_{13}+1-\mathrm{C}_{4} \mathrm{H}_{8}$ & $1.50 \times 10^{13}$ & 0 & 118.38 & 21,22 \\
\hline 12 & $2-\mathrm{C}_{10} \mathrm{H}_{21} \rightarrow 1-\mathrm{C}_{5} \mathrm{H}_{11}+1-\mathrm{C}_{5} \mathrm{H}_{10}$ & $1.50 \times 10^{13}$ & 0 & 118.38 & 21,22 \\
\hline 13 & $1-\mathrm{C}_{8} \mathrm{H}_{17} \rightleftharpoons 1-\mathrm{C}_{7} \mathrm{H}_{14}+\mathrm{CH}_{3}$ & $7.94 \times 10^{13}$ & 0 & 138.07 & 28 \\
\hline 14 & $1-\mathrm{C}_{8} \mathrm{H}_{17} \rightleftharpoons 1-\mathrm{C}_{6} \mathrm{H}_{13}+\mathrm{C}_{2} \mathrm{H}_{4}$ & $2.51 \times 10^{13}$ & 0 & 120.50 & 28 \\
\hline 15 & $1-\mathrm{C}_{8} \mathrm{H}_{17} \rightleftharpoons 1-\mathrm{C}_{5} \mathrm{H}_{11}+\mathrm{C}_{3} \mathrm{H}_{6}$ & $1.58 \times 10^{13}$ & 0 & 118.41 & 28 \\
\hline 16 & $1-\mathrm{C}_{8} \mathrm{H}_{17} \rightleftharpoons p-\mathrm{C}_{4} \mathrm{H}_{9}+1-\mathrm{C}_{4} \mathrm{H}_{8}$ & $5.01 \times 10^{12}$ & 0 & 121.75 & 28 \\
\hline 17 & $1-\mathrm{C}_{7} \mathrm{H}_{15} \rightarrow 1-\mathrm{C}_{6} \mathrm{H}_{12}+\mathrm{CH}_{3}$ & $2.00 \times 10^{13}$ & 0 & 129.67 & 21,22 \\
\hline 18 & $1-\mathrm{C}_{7} \mathrm{H}_{15} \rightarrow 1-\mathrm{C}_{5} \mathrm{H}_{10}+\mathrm{C}_{2} \mathrm{H}_{5}$ & $4.00 \times 10^{13}$ & 0 & 120.48 & 21,22 \\
\hline 19 & $1-\mathrm{C}_{7} \mathrm{H}_{15} \rightarrow p-\mathrm{C}_{4} \mathrm{H}_{9}+\mathrm{C}_{3} \mathrm{H}_{6}$ & $2.00 \times 10^{13}$ & 0 & 120.48 & 21,22 \\
\hline 20 & $1-\mathrm{C}_{7} \mathrm{H}_{15} \rightarrow 1-\mathrm{C}_{4} \mathrm{H}_{8}+n-\mathrm{C}_{3} \mathrm{H}_{7}$ & $2.00 \times 10^{13}$ & 0 & 120.48 & 21,22 \\
\hline 21 & $1-\mathrm{C}_{7} \mathrm{H}_{14} \rightarrow p-\mathrm{C}_{4} \mathrm{H}_{9}+\mathrm{C}_{3} \mathrm{H}_{5}$ & $3.16 \times 10^{16}$ & 0 & 338.83 & 21,22 \\
\hline 22 & $1-\mathrm{C}_{7} \mathrm{H}_{14}+\mathrm{H} \rightarrow 3 \mathrm{C}_{2} \mathrm{H}_{4}+\mathrm{CH}_{3}$ & $7.20 \times 10^{12}$ & 2.0 & 12.13 & 21,22 \\
\hline 23 & $1-\mathrm{C}_{7} \mathrm{H}_{14}+\mathrm{H} \rightarrow \mathrm{C}_{3} \mathrm{H}_{6}+\mathrm{C}_{2} \mathrm{H}_{5}+\mathrm{C}_{2} \mathrm{H}_{4}$ & $7.20 \times 10^{12}$ & 1.3 & 5.44 & 21,22 \\
\hline 24 & 1- $\mathrm{C}_{7} \mathrm{H}_{14}+\mathrm{C}_{2} \mathrm{H}_{5} \rightarrow \mathrm{C}_{2} \mathrm{H}_{6}+\mathrm{C}_{2} \mathrm{H}_{4}+\mathrm{C}_{4} \mathrm{H}_{6}+\mathrm{CH}_{3}$ & $1.40 \times 10^{1}$ & 3.5 & 18.13 & this work \\
\hline 25 & $1-\mathrm{C}_{6} \mathrm{H}_{13} \rightarrow p-\mathrm{C}_{4} \mathrm{H}_{9}+\mathrm{C}_{2} \mathrm{H}_{4}$ & $2.50 \times 10^{13}$ & 0 & 120.48 & 21,22 \\
\hline 26 & $1-\mathrm{C}_{6} \mathrm{H}_{13} \rightarrow n-\mathrm{C}_{3} \mathrm{H}_{7}+\mathrm{C}_{3} \mathrm{H}_{6}$ & $2.50 \times 10^{13}$ & 0 & 120.48 & 21,22 \\
\hline 27 & $1-\mathrm{C}_{6} \mathrm{H}_{12} \rightarrow n-\mathrm{C}_{3} \mathrm{H}_{7}+\mathrm{C}_{3} \mathrm{H}_{5}$ & $3.16 \times 10^{16}$ & 0 & 338.83 & 21,22 \\
\hline 28 & $1-\mathrm{C}_{6} \mathrm{H}_{12}+\mathrm{H} \rightarrow \mathrm{C}_{4} \mathrm{H}_{7}+\mathrm{C}_{2} \mathrm{H}_{4}+\mathrm{H}_{2}$ & $2.80 \times 10^{7}$ & 2.0 & 32.22 & 21.22 \\
\hline 29 & $1-\mathrm{C}_{6} \mathrm{H}_{12}+\mathrm{H} \rightarrow \mathrm{C}_{3} \mathrm{H}_{5}+\mathrm{C}_{3} \mathrm{H}_{6}+\mathrm{H}_{2}$ & $8.00 \times 10^{6}$ & 2.0 & 20.91 & 21,22 \\
\hline 30 & $1-\mathrm{C}_{6} \mathrm{H}_{12}+\mathrm{H} \rightarrow 1-\mathrm{C}_{4} \mathrm{H}_{8}+\mathrm{C}_{2} \mathrm{H}_{3}+\mathrm{H}_{2}$ & $8.00 \times 10^{6}$ & 2.0 & 20.91 & 21,22 \\
\hline 31 & $1-\mathrm{C}_{5} \mathrm{H}_{11} \rightarrow n-\mathrm{C}_{3} \mathrm{H}_{7}+\mathrm{C}_{2} \mathrm{H}_{4}$ & $2.50 \times 10^{13}$ & 0 & 120.48 & 21,22 \\
\hline 32 & $1-\mathrm{C}_{5} \mathrm{H}_{10} \rightarrow \mathrm{C}_{2} \mathrm{H}_{5}+\mathrm{C}_{3} \mathrm{H}_{5}$ & $3.16 \times 10^{16}$ & 0 & 338.83 & 21,22 \\
\hline 33 & $1-\mathrm{C}_{5} \mathrm{H}_{10}+\mathrm{H} \rightarrow \mathrm{C}_{5} \mathrm{H}_{9}+\mathrm{H}_{2}$ & $2.80 \times 10^{13}$ & 0 & 16.81 & 21,22 \\
\hline 34 & $1-\mathrm{C}_{5} \mathrm{H}_{10}+\mathrm{CH}_{3} \rightarrow \mathrm{C}_{5} \mathrm{H}_{9}+\mathrm{CH}_{4}$ & $1.00 \times 10^{11}$ & 0 & 30.62 & 21,22 \\
\hline 35 & $1-\mathrm{C}_{5} \mathrm{H}_{10} \rightarrow \mathrm{C}_{3} \mathrm{H}_{6}+\mathrm{C}_{2} \mathrm{H}_{2}+\mathrm{H}_{2}$ & $5.16 \times 10^{16}$ & 0 & 338.83 & this work \\
\hline 36 & $\mathrm{C}_{5} \mathrm{H}_{9} \rightarrow \mathrm{C}_{3} \mathrm{H}_{5}+\mathrm{C}_{2} \mathrm{H}_{4}$ & $2.50 \times 10^{13}$ & 0 & 125.68 & 21,22 \\
\hline 37 & $\mathrm{C}_{5} \mathrm{H}_{9} \rightarrow \mathrm{C}_{2} \mathrm{H}_{3}+\mathrm{C}_{3} \mathrm{H}_{6}$ & $2.50 \times 10^{13}$ & 0 & 125.68 & 21,22 \\
\hline 38 & $p-\mathrm{C}_{4} \mathrm{H}_{9} \rightleftharpoons \mathrm{C}_{2} \mathrm{H}_{5}+\mathrm{C}_{2} \mathrm{H}_{4}$ & $2.50 \times 10^{13}$ & 0 & 120.68 & 21,22 \\
\hline 39 & $p-\mathrm{C}_{4} \mathrm{H}_{9} \rightleftharpoons 1-\mathrm{C}_{4} \mathrm{H}_{8}+\mathrm{H}$ & $1.26 \times 10^{13}$ & 0 & 161.71 & 21,22 \\
\hline 40 & $1-\mathrm{C}_{4} \mathrm{H}_{8} \rightarrow \mathrm{C}_{2} \mathrm{H}_{3}+\mathrm{C}_{2} \mathrm{H}_{5}$ & $2.00 \times 10^{21}$ & -1.0 & 405.47 & 21,22 \\
\hline 41 & $1-\mathrm{C}_{4} \mathrm{H}_{8}+\mathrm{H} \rightleftharpoons \mathrm{C}_{4} \mathrm{H}_{7}+\mathrm{H}_{2}$ & $6.00 \times 10^{21}$ & 0 & 16.31 & 21,22 \\
\hline 42 & $1-\mathrm{C}_{4} \mathrm{H}_{8}+\mathrm{H} \rightleftharpoons \mathrm{H}_{2}+\mathrm{C}_{4} \mathrm{H}_{6}+\mathrm{H}$ & $2.15 \times 10^{8}$ & 2.0 & 10.41 & 31,32 \\
\hline 43 & $\mathrm{C}_{4} \mathrm{H}_{7}+\mathrm{C}_{2} \mathrm{H}_{5} \rightleftharpoons \mathrm{C}_{4} \mathrm{H}_{6}+\mathrm{C}_{2} \mathrm{H}_{6}$ & $4.00 \times 10^{12}$ & 0 & 0.00 & 21,22 \\
\hline 44 & $n-\mathrm{C}_{3} \mathrm{H}_{7} \rightleftharpoons \mathrm{CH}_{3}+\mathrm{C}_{2} \mathrm{H}_{4}$ & $1.60 \times 10^{14}$ & 0 & 129.89 & 21,22 \\
\hline 45 & $n-\mathrm{C}_{3} \mathrm{H}_{7} \rightleftharpoons \mathrm{H}+\mathrm{C}_{3} \mathrm{H}_{6}$ & $1.25 \times 10^{14}$ & 0 & 155.00 & 21,22 \\
\hline 46 & $i-\mathrm{C}_{3} \mathrm{H}_{7} \rightleftharpoons \mathrm{C}_{3} \mathrm{H}_{6}+\mathrm{H}$ & $6.30 \times 10^{13}$ & 0 & 154.60 & 21,22 \\
\hline 47 & $i-\mathrm{C}_{3} \mathrm{H}_{7} \rightleftharpoons \mathrm{C}_{2} \mathrm{H}_{4}+\mathrm{CH}_{3}$ & $2.00 \times 10^{10}$ & 0 & 123.58 & 21,22 \\
\hline 48 & $\mathrm{C}_{3} \mathrm{H}_{6} \rightarrow \mathrm{C}_{2} \mathrm{H}_{3}+\mathrm{CH}_{3}$ & $3.15 \times 10^{15}$ & 0 & 359.24 & 21,22 \\
\hline 49 & $\mathrm{C}_{3} \mathrm{H}_{6}+\mathrm{H} \rightleftharpoons \mathrm{C}_{3} \mathrm{H}_{5}+\mathrm{H}_{2}$ & $5.00 \times 10^{12}$ & 0 & 6.30 & 21,22 \\
\hline
\end{tabular}


continued Table 1

\begin{tabular}{|c|c|c|c|c|c|c|}
\hline No. & Reaction & & $A$ & $n$ & $E /\left(\mathrm{kJ} \cdot \mathrm{mol}^{-1}\right)$ & Ref. \\
\hline 50 & $\mathrm{C}_{3} \mathrm{H}_{6}+\mathrm{CH}_{3} \rightleftharpoons \mathrm{C}_{3} \mathrm{H}_{5}+\mathrm{CH}_{4}$ & & $8.96 \times 10^{12}$ & 0 & 35.62 & 21,22 \\
\hline 51 & $\mathrm{C}_{3} \mathrm{H}_{5}+\mathrm{H} \rightleftharpoons \mathrm{C}_{3} \mathrm{H}_{4}+\mathrm{H}_{2}$ & & $3.33 \times 10^{12}$ & 0 & 0.00 & 21,22 \\
\hline 52 & $\mathrm{C}_{3} \mathrm{H}_{4}+\mathrm{M} \rightarrow \mathrm{C}_{3} \mathrm{H}_{3}+\mathrm{H}+\mathrm{M}$ & & $2.00 \times 10^{18}$ & 0 & 334.62 & 21,22 \\
\hline 53 & $\mathrm{C}_{3} \mathrm{H}_{4}+\mathrm{H} \rightleftharpoons \mathrm{C}_{3} \mathrm{H}_{3}+\mathrm{H}_{2}$ & & $2.00 \times 10^{7}$ & 2.0 & 20.91 & 21,22 \\
\hline 54 & $\mathrm{C}_{3} \mathrm{H}_{4}+\mathrm{CH}_{3} \rightleftharpoons \mathrm{C}_{3} \mathrm{H}_{3}+\mathrm{CH}_{4}$ & & $2.00 \times 10^{11}$ & 0 & 32.22 & 21,22 \\
\hline 55 & $\mathrm{C}_{3} \mathrm{H}_{4}+\mathrm{H} \rightleftharpoons \mathrm{C}_{3} \mathrm{H}_{5}$ & & $2.00 \times 10^{12}$ & 0 & 11.31 & 21,22 \\
\hline 56 & $\mathrm{C}_{3} \mathrm{H}_{4} \rightleftharpoons p-\mathrm{C}_{3} \mathrm{H}_{4}$ & & $1.20 \times 10^{15}$ & 0 & 386.49 & 21,22 \\
\hline 57 & $p-\mathrm{C}_{3} \mathrm{H}_{4}+\mathrm{H} \rightleftharpoons \mathrm{C}_{2} \mathrm{H}_{2}+\mathrm{CH}_{3}$ & & $1.30 \times 10^{5}$ & 2.5 & 4.18 & 21,22 \\
\hline 58 & $p-\mathrm{C}_{3} \mathrm{H}_{4}+\mathrm{CH}_{3} \rightleftharpoons \mathrm{C}_{3} \mathrm{H}_{3}+\mathrm{CH}_{4}$ & & 1.50 & 3.5 & 23.42 & 21,22 \\
\hline 59 & $\mathrm{C}_{2} \mathrm{H}_{6}+\mathrm{H} \rightleftharpoons \mathrm{C}_{2} \mathrm{H}_{5}+\mathrm{H}_{2}$ & & $1.40 \times 10^{9}$ & 1.5 & 31.12 & 21,22 \\
\hline 60 & $\mathrm{C}_{2} \mathrm{H}_{6}+\mathrm{CH}_{3} \rightleftharpoons \mathrm{C}_{2} \mathrm{H}_{5}+\mathrm{CH}_{4}$ & & $1.50 \times 10^{-7}$ & 6.0 & 25.42 & 21,22 \\
\hline 61 & $\mathrm{C}_{2} \mathrm{H}_{4}+\mathrm{C}_{2} \mathrm{H}_{3} \rightleftharpoons \mathrm{C}_{4} \mathrm{H}_{6}+\mathrm{H}$ & & $2.80 \times 10^{21}$ & -2.44 & 6.16 & 21,22 \\
\hline 62 & $\mathrm{C}_{2} \mathrm{H}_{5}+\mathrm{CH}_{3} \rightleftharpoons \mathrm{C}_{2} \mathrm{H}_{4}+\mathrm{CH}_{4}$ & & $1.14 \times 10^{12}$ & 0 & 0.00 & 21,22 \\
\hline \multirow[t]{2}{*}{63} & $\mathrm{C}_{2} \mathrm{H}_{4}+\mathrm{H}+\mathrm{M} \rightarrow \mathrm{C}_{2} \mathrm{H}_{5}+\mathrm{M}$ & $k_{0}$ & $4.715 \times 10^{18}$ & 0 & 3.16 & 21,22 \\
\hline & & $k_{\infty}$ & $3.975 \times 10^{9}$ & 1.28 & 5.40 & 21,22 \\
\hline \multirow[t]{2}{*}{64} & $\mathrm{C}_{2} \mathrm{H}_{5}+\mathrm{M} \rightarrow \mathrm{C}_{2} \mathrm{H}_{4}+\mathrm{H}+\mathrm{M}$ & $k_{0}$ & $1.00 \times 10^{17}$ & 0 & 140.09 & 21,22 \\
\hline & & $k_{\infty}$ & $8.20 \times 10^{13}$ & 0 & 167.11 & 21,22 \\
\hline 65 & $\mathrm{C}_{2} \mathrm{H}_{4}+\mathrm{H} \rightleftharpoons \mathrm{C}_{2} \mathrm{H}_{3}+\mathrm{H}_{2}$ & & $5.40 \times 10^{14}$ & 0 & 62.44 & 21,22 \\
\hline 66 & $\mathrm{C}_{2} \mathrm{H}_{4}+\mathrm{M} \rightarrow \mathrm{C}_{2} \mathrm{H}_{3}+\mathrm{H}+\mathrm{M}$ & & $7.30 \times 10^{17}$ & 0 & 404.27 & 21,22 \\
\hline 67 & $\mathrm{C}_{2} \mathrm{H}_{4}+\mathrm{M} \rightleftharpoons \mathrm{C}_{2} \mathrm{H}_{2}+\mathrm{H}_{2}+\mathrm{M}$ & & $3.50 \times 10^{16}$ & 0 & 299.20 & 21,22 \\
\hline 68 & $\mathrm{C}_{2} \mathrm{H}_{3}+\mathrm{H} \rightleftharpoons \mathrm{C}_{2} \mathrm{H}_{2}+\mathrm{H}_{2}$ & & $1.20 \times 10^{13}$ & 0 & 0.00 & 21,22 \\
\hline \multirow[t]{2}{*}{69} & $\mathrm{C}_{2} \mathrm{H}_{3}+\mathrm{M} \rightleftharpoons \mathrm{C}_{2} \mathrm{H}_{2}+\mathrm{H}+\mathrm{M}$ & $k_{0}$ & $4.153 \times 10^{41}$ & -7.5 & 190.53 & 21,22 \\
\hline & & $k_{\infty}$ & $2.00 \times 10^{14}$ & 0 & 166.40 & 21,22 \\
\hline 70 & $\mathrm{CH}_{4}+\mathrm{H} \rightleftharpoons \mathrm{CH}_{3}+\mathrm{H}_{2}$ & & $1.30 \times 10^{4}$ & 3.0 & 33.62 & 21,22 \\
\hline \multirow[t]{2}{*}{71} & $\mathrm{CH}_{3}+\mathrm{H}+\mathrm{M} \rightleftharpoons \mathrm{CH}_{4}+\mathrm{M}$ & $k_{0}$ & $6.257 \times 10^{23}$ & -1.8 & 0.00 & 21,22 \\
\hline & & $k_{\infty}$ & $2.108 \times 10^{14}$ & 0 & 0.00 & 21,22 \\
\hline 72 & $2 \mathrm{CH}_{3} \rightarrow \mathrm{C}_{2} \mathrm{H}_{4}+\mathrm{H}_{2}$ & & $1.00 \times 10^{14}$ & 0 & 134.09 & 21,22 \\
\hline \multirow[t]{2}{*}{73} & $\mathrm{CH}_{3}+\mathrm{CH}_{3}+\mathrm{M} \rightleftharpoons \mathrm{C}_{2} \mathrm{H}_{6}+\mathrm{M}$ & $k_{0}$ & $1.77 \times 10^{50}$ & -9.67 & 26.04 & 32 \\
\hline & & $k_{\infty}$ & $2.12 \times 10^{22}$ & -0.97 & 0.00 & 32 \\
\hline 74 & $2 \mathrm{CH}_{3} \rightleftharpoons \mathrm{C}_{2} \mathrm{H}_{5}+\mathrm{H}$ & & $5.65 \times 10^{11}$ & 0 & 61.44 & 21,22 \\
\hline 75 & $2 \mathrm{H}+\mathrm{M} \rightleftharpoons \mathrm{H}_{2}+\mathrm{M}$ & & $1.865 \times 10^{18}$ & -1.0 & 0.00 & 21,22 \\
\hline
\end{tabular}

(a) Rate constants are written as $A T \exp (-E / R T)$, the units of $A$ are mol, $\mathrm{cm}^{3}, \mathrm{~s}, \mathrm{~kJ}, \mathrm{~K}$. (b) Third-body coefficients are $[\mathrm{M}]=0.4\left[\mathrm{~N}_{2}\right]+3.0\left[\mathrm{CH}_{4}\right]+$ $3.0\left[\mathrm{C}_{10} \mathrm{H}_{22}\right]$; (c) For those rate constants $k$ that depend on pressure, $k_{0}$ and $k_{\infty}$ are given in the table. The Troe formula is: $k=\frac{k_{0} k_{\infty}[\mathrm{M}]}{k_{\infty}+k_{0}[\mathrm{M}]} F$, where $k_{0}$ is the rate constant for the low-pressure limit, $k_{\infty}$ is the rate constant for the high pressure limit, [M] is the third-body coefficients, $F$ is the blending function.

$$
\left.\lg F=\frac{\lg F_{\text {cent }}}{1.0+\left[\frac{\lg \frac{k_{0}[\mathrm{M}]}{k_{\infty}}-0.4-0.67 \times \log F_{\text {cent }}}{0.75-1.27 \times \lg F_{\text {cent }}-0.14 \times\left(\lg \frac{k_{0}[\mathrm{M}]}{k_{\infty}}-0.4-0.67 \times \lg F_{\text {cent }}\right)}\right]}\right]
$$

Broadening functions $\left(F_{\text {cent }}\right)$ are, R63: $F_{\text {cent }}=0.24 \times \exp (-T / 40.0)+0.76 \times \exp (-T / 1025.0) ; \mathrm{R} 64: F_{\text {cent }}=0.25 \times \exp (-T / 97.0)+0.75 \times$ $\exp (-T / 1379.0) ; \mathrm{R} 69: F_{\text {cen }}=0.35 ; \mathrm{R} 71: F_{\text {cenl }}=0.577 \times \exp (-T / 2370.0) ; \mathrm{R} 73: F_{\text {cenl }}=0.5325 \times \exp (-T / 151.0)+0.4675 \times \exp (-T / 1038.0)$

$\mathrm{C}_{4} \mathrm{H}_{8}$ 生成量的预测都偏低, 尤其是 Lawrence 机理对 $\mathrm{C}_{4} \mathrm{H}_{8}$ 的预测则偏低两倍以上, 由于 Mech33 机理增 加了关于 $\mathrm{C}_{4} \mathrm{H}_{8}$ 的重要反应 $\mathrm{C}_{4} \mathrm{H}_{8}+\mathrm{H} \rightleftharpoons \mathrm{H}_{2}+\mathrm{C}_{4} \mathrm{H}_{6}+\mathrm{H},{ }^{31}$ 因而准确地预测了 $\mathrm{C}_{4} \mathrm{H}_{8}$ 产量随停留时间的变化. 从 图 1 的对比中, 可以看到本文构建的正癸烷热裂解 机理无论是预测正癸烷的转化, 还是预测其主要分 解产物分布, 都与实验结果非常吻合.

\section{2 激波管}

为了在较宽的温度范围内验证本文机理是否 能够较好地描述正癸烷的裂解过程, 我们采用 CHEMKIN 零维均质反应模型 ${ }^{33}$ 对正癸烷裂解过程 进行了数值模拟. 模拟条件如下: 温度 900-1800 K, 反应时间 1.27-1.90 ms, 压力 4.73-7.52 MPa, 正癸 烷在稀释其体 $\mathrm{N}_{2}$ 中的摩尔分数为 $1.0725 \times 10^{-4}$. 图 2 给出了上述计算结果与 Malewicki 和 Brezinsky ${ }^{17}$ 激 波管实验结果的对比, 同时还给出了 Lawrence机理 ${ }^{12}$ 

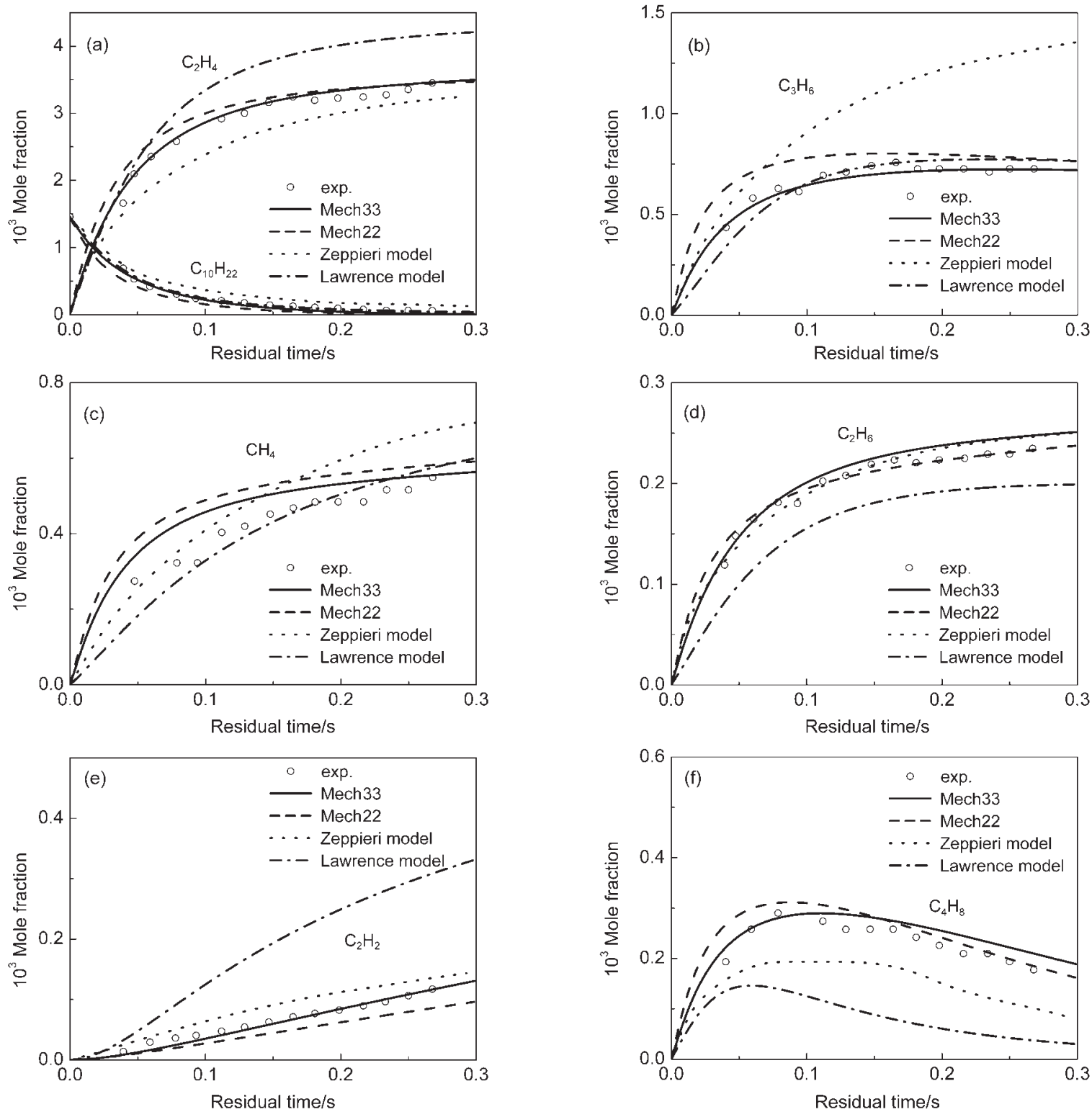

图 1 采用本文机理(Mech33 和 Mech22), Zeppieri机理, ${ }^{10}$ Lawrence机理 ${ }^{12}$ 计算得到的流动反应器中 正癸烷裂解产物分布与实验结果 ${ }^{10}$ 的对比

Fig.1 Comparison of experimental ${ }^{10}$ and computed species profiles using the present model (Mech33 and Mech22), Zeppieri model, ${ }^{10}$ and Lawrence model ${ }^{12}$ during $\boldsymbol{n}$-decane pyrolysis in a flow reactor $p=0.1 \mathrm{MPa}, T=1060 \mathrm{~K}$, initial mole fraction of $n$-decane is $1.456 \times 10^{-3}$.

的计算结果. 从图 2(a)中可以看出, 本文构建的机理 和 Lawrence机理 ${ }^{12}$ 都能够较准确地描述各个温度下 正癸烷的裂解转化量. 本文机理在 1200-1600 K温 度范围内对于 $\mathrm{C}_{2} \mathrm{H}_{4}$ 和 $\mathrm{C}_{2} \mathrm{H}_{2}$ 的预测虽然总体上与实 验结果符合较好, 但与实验值仍有一些差别. 此外, 可以看出随着温度的升高, 乙烯产量先升后降, 而 乙炔的产量则一直在升高, 研究表明, ${ }^{34}$ 正癸烷热解 过程中产生的不饱和烯烃和炔烃越多, 对应的化学 吸热量越大, 因此提高温度可以有助于提高正癸烷 裂解的化学热沉. 图 2(b)表明, 本文机理对于 $\mathrm{C}_{2} \mathrm{H}_{6}$ 的
预测在 1200-1400 K 范围内偏高, 而 Lawrence 机 理 ${ }^{12}$ 则在更宽的温度范围内低估了 $\mathrm{C}_{2} \mathrm{H}_{6}$ 的产量; 本 文机理对 $p-\mathrm{C}_{3} \mathrm{H}_{4}$ 的产量的预测与实验值符合较好, 而 Lawrence机理 ${ }^{12}$ 在整个计算温度范围内的计算结 果与实验结果差别较大. 如图 2(c) 所示, 在 1200$1300 \mathrm{~K}$ 温度范围内, 本文机理对 $\mathrm{C}_{3} \mathrm{H}_{6}$ 的预测比实验 结果略高, 但在温度高于 $1300 \mathrm{~K}$ 以后, 本文机理很 好地预报了实验值结果, 且好于 Lawrence 机理 ${ }^{12}$ 的 预测结果; 本文机理在整个温度范围内预测的 $\mathrm{C}_{4} \mathrm{H}_{8}$ 与实验值符合较好, 进一步说明了, 本文机理增加 

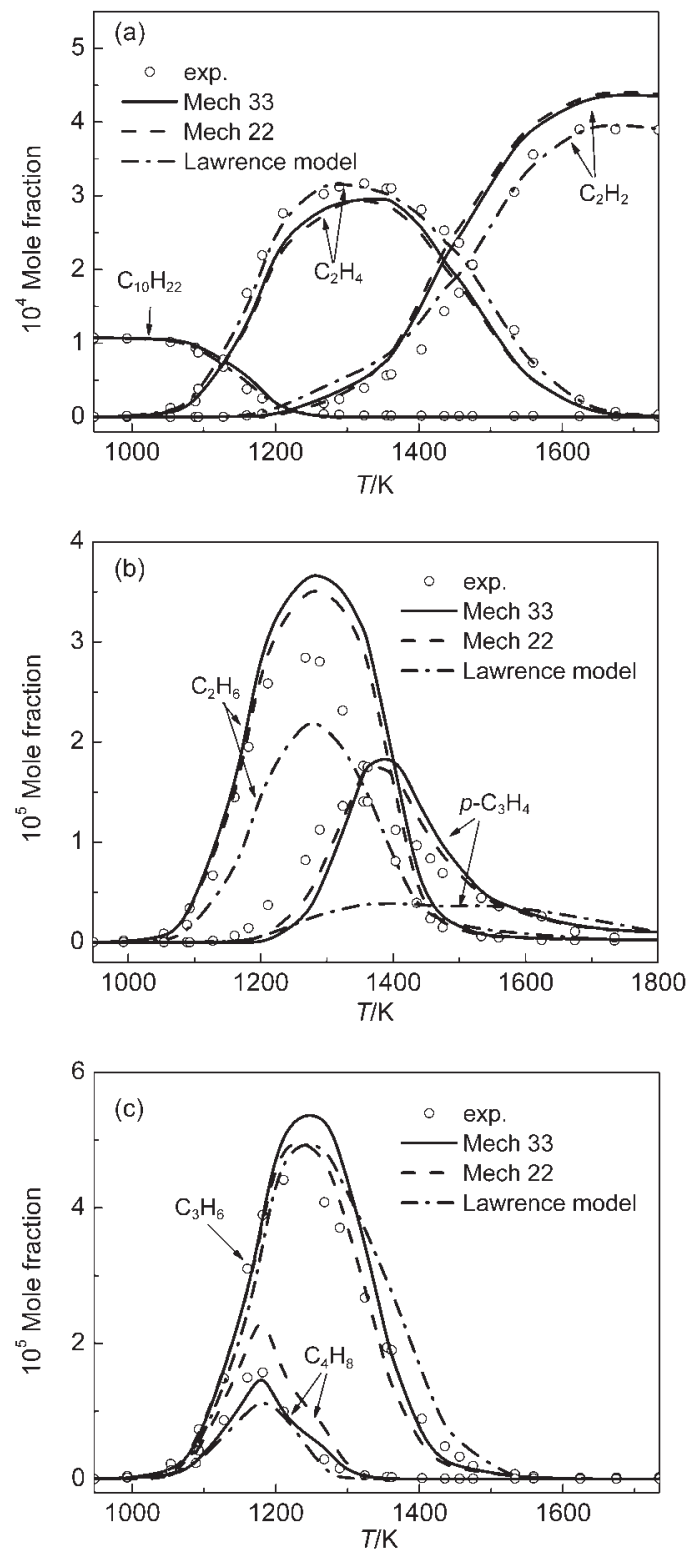

图 2 采用本文机理(Mech33 和 Mech22)和 Lawrence 机理 ${ }^{12}$ 计算得到的激波管中正癸烷热解组分摩尔分数随温 度的变化与实验结果 ${ }^{17}$ 的对比

Fig.2 Comparison of experimental ${ }^{17}$ and computed species mole fraction variation with temperature during $n$-decane pyrolysis in a shock tube using the present model

(Mech33 and Mech22) and Lawrence model ${ }^{12}$

$p=4.73-7.52 \mathrm{MPa}, T=900-1800 \mathrm{~K}$, initial mole fraction of $n$-decane is $1.0725 \times 10^{-4}$.

关于 $\mathrm{C}_{4} \mathrm{H}_{8}$ 的重要反应 $\mathrm{C}_{4} \mathrm{H}_{8}+\mathrm{H} \rightleftharpoons \mathrm{H}_{2}+\mathrm{C}_{4} \mathrm{H}_{6}+\mathrm{H}^{31}$ 对准确 描述 $\mathrm{C}_{4} \mathrm{H}_{8}$ 的变化过程是必要的.

可见, 本文构建的正癸烷热裂解机理(Mech33) 对 $1060 \mathrm{~K}$ 温度下的流动反应器和 900-1800 K温度 范围内的激波管中正癸烷裂解过程及其主要热裂 解组分浓度分布的预测与实验结果较吻合, 且多数
情况下明显好于 Zeppieri 机理 ${ }^{10}$ 和 Lawrence 机理 ${ }^{12}$ 计算结果, 而且本文机理组分数和反应数大大少于 这两个机理, 因而在数值模拟中可以节省计算资 源, 提高计算效率.

\section{4 正癸烷裂热解机理的进一步简化}

上述 Mech33 机理相对于现有的正癸烷热裂解 机理规模已经很小了, 但是为了考虑到今后在多维 CFD 数值模拟中应用, 我们尝试在此机理的基础上 进行进一步的简化, 期望得到更加适用于工程应用 的动力学机理模型. 为此, 我们对 Mech33 机理模型 进行了动力学分析.

灵敏度分析 ${ }^{35}$ 是分析反应机理的重要方法, 用

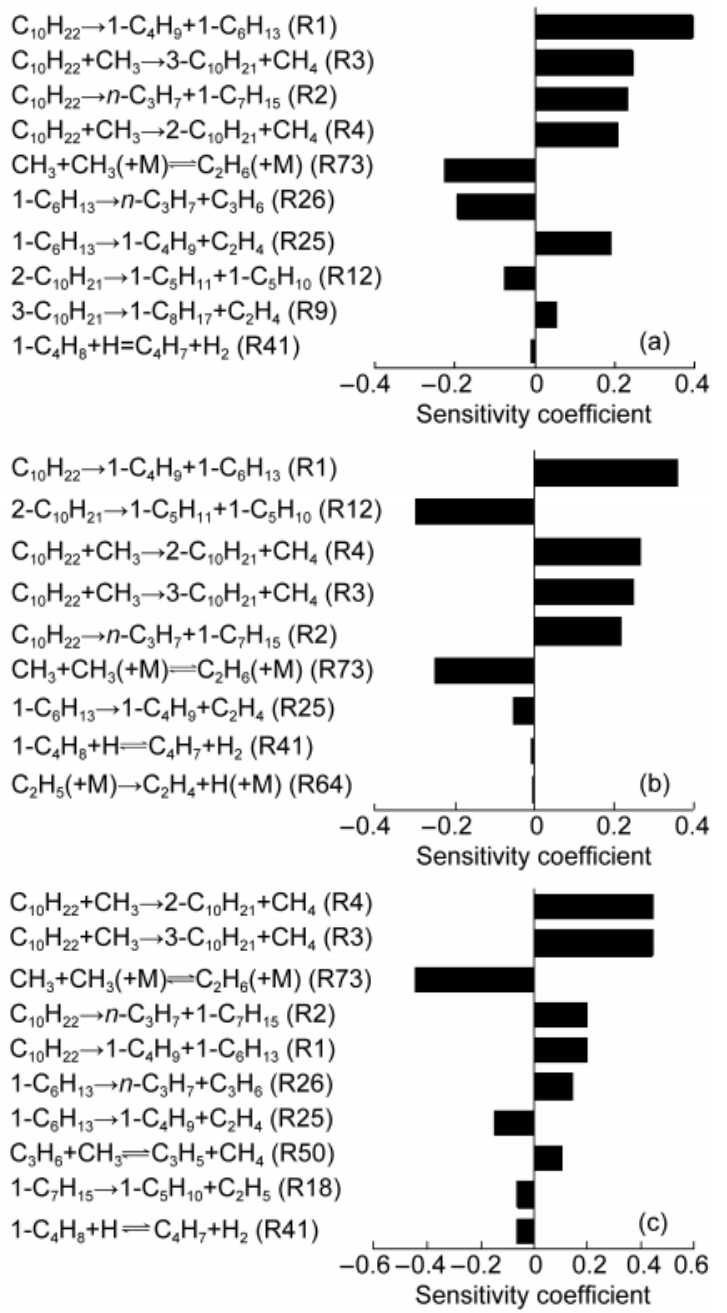

图 3 流动反应器中正癸烷裂解主要产物 $\mathrm{C}_{2} \mathrm{H}_{4}(\mathrm{a}) 、 \mathrm{C}_{3} \mathrm{H}_{6}$ (b) 和 $\mathrm{CH}_{4}$ (c) 对反应的灵敏度系数

Fig.3 Sensitivity coefficient of $\mathrm{C}_{2} \mathrm{H}_{4}(\mathrm{a}), \mathrm{C}_{3} \mathrm{H}_{6}$ (b), and

$\mathrm{CH}_{4}$ (c) to reaction rate parameters during $n$-decane pyrolysis in a flow reactor

$p=0.1 \mathrm{MPa}, T=1060 \mathrm{~K}$, initial mole fraction of $n$-decane is $1.456 \times 10^{-3}$. 
表 2 正癸烷热裂解简化机理(Mech22)部分反应的动力学参数

Table 2 Part of rate parameters of reactions employed in the simplfied model (Mech 22) of $n$-decane pyrolysis

\begin{tabular}{|c|c|c|c|c|}
\hline No. & Reaction & $A$ & $n$ & $E /\left(\mathrm{kJ} \cdot \mathrm{mol}^{-1}\right)$ \\
\hline 1 & $n-\mathrm{C}_{10} \mathrm{H}_{22} \rightarrow 2 \mathrm{C}_{2} \mathrm{H}_{5}+3 \mathrm{C}_{2} \mathrm{H}_{4}$ & $6.55 \times 10^{16}$ & 0.0 & 348.26 \\
\hline 2 & $n-\mathrm{C}_{10} \mathrm{H}_{22} \rightarrow \mathrm{C}_{2} \mathrm{H}_{5}+\mathrm{C}_{2} \mathrm{H}_{4}+n-\mathrm{C}_{3} \mathrm{H}_{7}+\mathrm{C}_{3} \mathrm{H}_{6}$ & $6.55 \times 10^{16}$ & 0.0 & 348.26 \\
\hline 3 & $n-\mathrm{C}_{10} \mathrm{H}_{22} \rightarrow n-\mathrm{C}_{3} \mathrm{H}_{7}+1-\mathrm{C}_{5} \mathrm{H}_{10}+\mathrm{C}_{2} \mathrm{H}_{5}$ & $1.31 \times 10^{17}$ & 0.0 & 349.18 \\
\hline 4 & $n-\mathrm{C}_{10} \mathrm{H}_{22} \rightarrow 2 n-\mathrm{C}_{3} \mathrm{H}_{7}+1-\mathrm{C}_{4} \mathrm{H}_{8}$ & $1.31 \times 10^{17}$ & 0.0 & 349.18 \\
\hline 5 & $n-\mathrm{C}_{10} \mathrm{H}_{22}+\mathrm{CH}_{3} \rightarrow \mathrm{C}_{2} \mathrm{H}_{5}+2 \mathrm{C}_{2} \mathrm{H}_{4}+1-\mathrm{C}_{4} \mathrm{H}_{8}+\mathrm{CH}_{4}$ & $6.80 \times 10^{12}$ & 0.0 & 35.97 \\
\hline 6 & $n-\mathrm{C}_{10} \mathrm{H}_{22}+\mathrm{CH}_{3} \rightarrow n-\mathrm{C}_{3} \mathrm{H}_{7}+\mathrm{C}_{3} \mathrm{H}_{6}+1-\mathrm{C}_{4} \mathrm{H}_{8}+\mathrm{CH}_{4}$ & $8.10 \times 10^{12}$ & 0.0 & 35.97 \\
\hline 7 & $n-\mathrm{C}_{10} \mathrm{H}_{22}+\mathrm{CH}_{3} \rightarrow \mathrm{C}_{2} \mathrm{H}_{5}+4 \mathrm{C}_{2} \mathrm{H}_{4}+\mathrm{CH}_{4}$ & $3.40 \times 10^{12}$ & 0.0 & 35.97 \\
\hline 8 & $n-\mathrm{C}_{10} \mathrm{H}_{22}+\mathrm{CH}_{3} \rightarrow n-\mathrm{C}_{3} \mathrm{H}_{7}+\mathrm{C}_{3} \mathrm{H}_{6}+2 \mathrm{C}_{2} \mathrm{H}_{4}+\mathrm{CH}_{4}$ & $6.80 \times 10^{12}$ & 0.0 & 35.97 \\
\hline 9 & $n-\mathrm{C}_{10} \mathrm{H}_{22}+\mathrm{CH}_{3} \rightarrow \mathrm{C}_{3} \mathrm{H}_{5}+\mathrm{C}_{2} \mathrm{H}_{5}+2 \mathrm{C}_{2} \mathrm{H}_{4}+\mathrm{CH}_{3}+\mathrm{CH}_{4}$ & $3.40 \times 10^{12}$ & 0.0 & 35.97 \\
\hline 10 & $n-\mathrm{C}_{10} \mathrm{H}_{22}+\mathrm{CH}_{3} \rightarrow \mathrm{C}_{2} \mathrm{H}_{5}+\mathrm{C}_{3} \mathrm{H}_{6}+1-\mathrm{C}_{5} \mathrm{H}_{10}+\mathrm{CH}_{4}$ & $1.97 \times 10^{13}$ & 0.0 & 35.97 \\
\hline 11 & $n-\mathrm{C}_{10} \mathrm{H}_{22}+\mathrm{CH}_{3} \rightarrow 2 \mathrm{C}_{3} \mathrm{H}_{6}+\mathrm{C}_{2} \mathrm{H}_{5}+\mathrm{C}_{2} \mathrm{H}_{4}+\mathrm{CH}_{4}$ & $4.70 \times 10^{12}$ & 0.0 & 35.97 \\
\hline 12 & $n-\mathrm{C}_{10} \mathrm{H}_{22}+\mathrm{CH}_{3} \rightarrow \mathrm{CH}_{3}+\mathrm{C}_{3} \mathrm{H}_{6}+n-\mathrm{C}_{3} \mathrm{H}_{7}+\mathrm{C}_{3} \mathrm{H}_{5}+\mathrm{CH}_{4}$ & $4.70 \times 10^{12}$ & 0.0 & 35.97 \\
\hline 13 & $n-\mathrm{C}_{10} \mathrm{H}_{22}+\mathrm{CH}_{3} \rightarrow n-\mathrm{C}_{3} \mathrm{H}_{7}+\mathrm{C}_{2} \mathrm{H}_{4}+1-\mathrm{C}_{5} \mathrm{H}_{10}+\mathrm{CH}_{4}$ & $4.70 \times 10^{12}$ & 0.0 & 35.97 \\
\hline 14 & $n-\mathrm{C}_{10} \mathrm{H}_{22}+\mathrm{H} \rightarrow \mathrm{C}_{2} \mathrm{H}_{5}+2 \mathrm{C}_{2} \mathrm{H}_{4}+1-\mathrm{C}_{4} \mathrm{H}_{8}+\mathrm{H}_{2}$ & $8.00 \times 10^{8}$ & 2.0 & 20.91 \\
\hline 15 & $n-\mathrm{C}_{10} \mathrm{H}_{22}+\mathrm{H} \rightarrow n-\mathrm{C}_{3} \mathrm{H}_{7}+\mathrm{C}_{3} \mathrm{H}_{6}+1-\mathrm{C}_{4} \mathrm{H}_{8}+\mathrm{H}_{2}$ & $4.10 \times 10^{8}$ & 2.0 & 20.91 \\
\hline 16 & $n-\mathrm{C}_{10} \mathrm{H}_{22}+\mathrm{H} \rightarrow \mathrm{C}_{2} \mathrm{H}_{5}+4 \mathrm{C}_{2} \mathrm{H}_{4}+\mathrm{H}_{2}$ & $1.50 \times 10^{8}$ & 2.0 & 20.91 \\
\hline 17 & $n-\mathrm{C}_{10} \mathrm{H}_{22}+\mathrm{H} \rightarrow n-\mathrm{C}_{3} \mathrm{H}_{7}+\mathrm{C}_{3} \mathrm{H}_{6}+2 \mathrm{C}_{2} \mathrm{H}_{4}+\mathrm{H}_{2}$ & $3.00 \times 10^{8}$ & 2.0 & 20.91 \\
\hline 18 & $n-\mathrm{C}_{10} \mathrm{H}_{22}+\mathrm{H} \rightarrow \mathrm{C}_{3} \mathrm{H}_{5}+\mathrm{C}_{2} \mathrm{H}_{5}+2 \mathrm{C}_{2} \mathrm{H}_{4}+\mathrm{CH}_{3}+\mathrm{H}_{2}$ & $1.50 \times 10^{8}$ & 2.0 & 20.91 \\
\hline 19 & $n-\mathrm{C}_{10} \mathrm{H}_{22}+\mathrm{H} \rightarrow 2 \mathrm{C}_{3} \mathrm{H}_{6}+\mathrm{C}_{2} \mathrm{H}_{5}+\mathrm{C}_{2} \mathrm{H}_{4}+\mathrm{H}_{2}$ & $2.60 \times 10^{8}$ & 2.0 & 20.91 \\
\hline 20 & $n-\mathrm{C}_{10} \mathrm{H}_{22}+\mathrm{H} \rightarrow \mathrm{CH}_{3}+\mathrm{C}_{3} \mathrm{H}_{6}+n-\mathrm{C}_{3} \mathrm{H}_{7}+\mathrm{C}_{3} \mathrm{H}_{5}+\mathrm{H}_{2}$ & $2.60 \times 10^{8}$ & 2.0 & 20.91 \\
\hline 21 & $n-\mathrm{C}_{10} \mathrm{H}_{22}+\mathrm{H} \rightarrow n-\mathrm{C}_{3} \mathrm{H}_{7}+\mathrm{C}_{2} \mathrm{H}_{4}+1-\mathrm{C}_{5} \mathrm{H}_{10}+\mathrm{H}_{2}$ & $2.60 \times 10^{8}$ & 2.0 & 20.91 \\
\hline 22 & $1-\mathrm{C}_{5} \mathrm{H}_{10} \rightleftharpoons \mathrm{C}_{2} \mathrm{H}_{5}+\mathrm{C}_{3} \mathrm{H}_{5}$ & $3.16 \times 10^{16}$ & 0.0 & 338.83 \\
\hline 23 & $1-\mathrm{C}_{5} \mathrm{H}_{10} \rightarrow \mathrm{C}_{3} \mathrm{H}_{6}+\mathrm{C}_{2} \mathrm{H}_{2}+\mathrm{H}_{2}$ & $5.16 \times 10^{16}$ & 0.0 & 338.83 \\
\hline
\end{tabular}

Rate constants are written as $A T^{n} \exp (-E / R T)$, the units of $A$ are $\mathrm{mol}, \mathrm{cm}^{3}, \mathrm{~s}, \mathrm{~kJ}, \mathrm{~K}$.

来识别对某个重要目标函数的速率控制反应步, 灵 敏度系数的大小反映了基元反应速率常数对目标 函数的影响. 对于许多基元反应, 速率常数的变化 几乎对目标函数没有影响, 此时准稳态和局部平衡 假设是适用的, 满足这些条件的反应可以被取消; 对一些灵敏度系数比较大的基元反应, 速率常数的 变化对目标函数有很大影响, 这些反应在整个反应 过程中是重要的, 而且是速率控制步. 图 3 给出了温 度 $T=1060 \mathrm{~K}$ 条件下流动反应器中正癸烷热解转化 率 $20 \%$ 处的对主要产物 $\left(\mathrm{CH}_{4} 、 \mathrm{C}_{2} \mathrm{H}_{4} 、 \mathrm{C}_{3} \mathrm{H}_{6}\right)$ 的产率相 对灵敏度系数最大的十个基元反应. 灵敏度系数为 正表示该反应速率常数的增加对产物产率有正的 影响, 灵敏度系数为负则表示该反应速率常数的增 加对产物产率有负的影响.

由图 3(a)可以看出, 对乙烯产率灵敏度系数最 大的 4 个反应是正癸烷 $\mathrm{C}-\mathrm{C}$ 键断裂反应以及正癸 烷脱氢反应. 同样, 从图 3(b)和 3(c) 中可以看出, 正 癸烷的分解反应和脱氢反应对丙烯和甲烷产率的 影响很大, 因此, 正癸烷 $\mathrm{C}-\mathrm{C}$ 键断裂反应和有自由 基参与的脱氢反应是影响最终产物分布的速率控 制步, 而正癸烷或癸烷基分解产生的中间组分如
$\mathrm{C}_{8} \mathrm{H}_{17} 、 \mathrm{C}_{7} \mathrm{H}_{15}$ 等的进一步分解反应速率非常快, 属于 准稳态组分, 对最终产物的生成影响不大.

通过灵敏度分析可知, 正癸烷热解过程中通过 与 $\mathrm{H}$ 和 $\mathrm{CH}_{3}$ 作用进行脱氢反应以及正癸烷 $\mathrm{C}-\mathrm{C}$ 键 断裂反应是整个热解过程的速率控制步, 癸烷基 $\beta$ 键断裂反应以及进一步发生的烷基和烯烃的分解 反应都可认为是速率无限快的反应. ${ }^{13,17}$ 根据局部平 衡和局部稳态假设, 我们考虑合并相应的反应, 从 而实现对 Mech33 机理的简化, 得到正癸烷通过脱 氢和热解反应直接生成小分子烷烃和烯烃以及自 由基的更简单的机理. 反应 $\mathrm{R} 1-\mathrm{R} 4$ 是合并了正癸烷 $\mathrm{C}-\mathrm{C}$ 键断裂反应和接下来发生的分解反应，直接 由正癸烷裂解生成小分子化合物. 由于正癸烷 $\mathrm{C}-$ $\mathrm{C}$ 键断裂反应是速率控制步, 其速率常数采用正癸 烷 $\mathrm{C}-\mathrm{C}$ 键断裂反应的速率常数. 反应 $\mathrm{R} 5-\mathrm{R} 13$ 以及 反应 $\mathrm{R} 15-\mathrm{R} 21$ 分别合并了自由基 $\mathrm{CH}_{3}$ 和 $\mathrm{H}$ 作用下 正癸烷脱氢反应和接下发生的 $\beta$-键断裂反应, 速率 常数采用脱氢反应的速率常数. 由于删除了一些中 间组分和相应的反应, 因此需要对简化后机理中的 速率常数做微调整, 调整后的子模型(如表 2 所示)和 $\mathrm{C}_{0}-\mathrm{C}_{4}$ (表 1 中 $\mathrm{R} 40-\mathrm{R} 75$ ) 机理一起, 构成了仅包含 22 
种组分和 59 个反应的正癸烷热裂解机理(Mech22).

为了验证 Mech22 机理的合理性, 我们使用 Mech22机理对相同条件下的正癸烷热裂解过程进 行了重新计算. 同样采用 CHEMKIN ${ }^{33}$ 管流反应器模 型, 计算条件为: 压力 $p=0.1 \mathrm{MPa}$, 温度 $T=1060 \mathrm{~K}$, 停 留时间 $\tau=0.3 \mathrm{~s}$, 正癸烷在稀释其体 $\mathrm{N}_{2}$ 中摩尔分数 $1.456 \times 10^{-3}$. 计算结果如图 2 所示. 由图可见, Mech22 机理对正癸烷及其主要的热裂解产物 $\mathrm{C}_{2} \mathrm{H}_{4} 、 \mathrm{C}_{3} \mathrm{H}_{6}$ 、 $\mathrm{CH}_{4} 、 \mathrm{C}_{2} \mathrm{H}_{6} 、 \mathrm{C}_{2} \mathrm{H}_{2}$ 和 $\mathrm{C}_{4} \mathrm{H}_{8}$ 的预测与 $\mathrm{Mech} 33$ 机理以及 实验结果均吻合较好. 由于简化机理(Mech22)忽略 了许多中间反应步骤, 虽然这些反应对热解过程影 响较小, 但还是对正癸烷的热裂解进程有一定的影 响, 由图可见, 在 Mech22 机理中正癸烷的热解过程 略微加快, 燃料的消耗和组分的生成较 Mech33 机 理更加的迅速, 但是总体上与 Mech33 机理符合较 好, 表明机理的简化是有效的, 而且准确性较高.

同样, 采用 Mech22 机理在 CHEMKIN 零维均质 反应模型 ${ }^{33}$ 中对温度 900-1800 K, 反应时间 1.27$1.90 \mathrm{~ms}$, 压力 4.73-7.52 $\mathrm{MPa}$, 正癸烷在稀释气体 $\mathrm{N}_{2}$ 中的摩尔分数为 $1.0725 \times 10^{-4}$ 条件下的正癸烷裂解 过程进行了数值模拟. 计算结果如图 3 所示. 由图可 见, Mech22 能够与 Mech33 机理以及实验结果吻合 较好, 表明在较宽的温度范围内该简化机理能够较 好地再现源机理. 简化后得到的动力学机理模型 (Mech22)与 Mech33 机理相比, 减少了组分数, 因此 对于减少 CFD 工程应用中的计算量更具有实际的 意义.

\section{5 结 论}

本文通过分析正癸烷裂解过程中的主要反应 类型和反应路径, 构建了一个包含 33 种组分和 77 个反应的正癸烷热裂解动力学机理模型(Mech33), 该模型能够很好地描述正癸烷热裂解过程, 准确预 测热裂解主要产物分布. 在 Mech33 机理的基础上 通过灵敏度分析, 找出了对主要产物生成影响较大 的速率控制步, 采用稳态假设, 通过合并相关的反 应得到 22 种组分和 59 步反应的简化机理(Mech22), 该机理对主要热裂解组分的预测与 Mech33 机理和 实验结果非常吻合, 验证了该简化方法的有效性. 本文得到的两个机理都具有较少的组分数和反应 数, 规模小, 为实现耦合化学反应的工程计算提供 了有价值的动力学机理模型.

\section{References}

(1) Emeric, D.; Marc, B.; Olivier, H.; Marie, P. M.; Gascoin, N.; Gillard, P. Fuel Reforming for Scramjet Thermal Management and Combustion Optimization. AIAA/CIRA 13th International Space Planes and Hypersonics Systems and Technologies Conference, Capua, Italy, May 16-20, 2005.

(2) Falempin, F.; Bouchez, M.; Salmon, T.; Lespade, P.; Avrashkov, V. An Innovative Technology for Fuel-Cooled Composite Materials Structure. AIAA/NAL-NASDA-ISAS 10th International Space Planes and Hypersonic Systems and Technologies Conference, Kyoto, Japan, April 24-27, 2001.

(3) Emeric, D.; Marc, B.; Roda, B.; Battin, F. L.; Marie, P. M.; René, F. Contribution to Scramjet Active Cooling Analysis Using N-dodecane Decomposition Model. 12th AIAA International Space Planes and Hypersonic Systems and Technologies Conference, Norfolk, Virginia, America, December 15-19, 2003.

(4) Huang, H.; Sobel, D. R.; Spadaccini, L. J. Endothermic Heat-Sink of Hydrocarbon Fuels for Scramjet Cooling. 38th AIAA/ASME/SAE/ASEE Joint Propulsion Conference \& Exhibit, Indianapolis, Indiana, America, July 7-11, 2002.

(5) Dagaut, P.; Reuillon, M.; Boettner, J. C.; Cathonnet, M. Symp. Int. Combust. 1994, 25 (1), 919. doi: 10.1016/S0082-0784(06) 80727-7

(6) Dagaut, P.; Bakali, E. A.; Ristori, A. Fuel 2006, 85 (7-8), 944.

(7) Humer, S.; Frassoldati, A.; Granata, S.; Faravelli, T.; Ranzi, E.; Seiser, R.; Seshadri, K. Proc. Combust. Inst. 2007, 31 (1), 393. doi: 10.1016/j.proci.2006.08.008

(8) Yu, J.; Eser, S. Ind. Eng. Chem. Res. 1997, 36 (3), 585. doi: 10.1021/ie9603934

(9) Jiao, Y.; Li, J.; Wang, J. B.; Wang, J. L.; Zhu, Q.; Chen, Y. Q.; Li, X. Y. Acta Phys. -Chim. Sin. 2011, 27, 1061. [焦 毅, 李 军, 王静波, 王健礼, 朱 权, 陈耀强, 李象远. 物理化学学 报, 2011, 27, 1061.] doi: 10.3866/PKU.WHXB20110437

(10) Zeppieri, S. P.; Klotz, S. D.; Dryer, F. L. Proc. Combust. Inst. 2000, 28 (2), 1587. doi: 10.1016/S0082-0784(00)80556-1

(11) Zhao, Z. W.; Li, J.; Kazakov, A.; Dryer, F. L.; Zeppieri, S. P. Combust. Sci. Tech. 2005, 177 (1), 89.

(12) Westbrook, C. K.; Pitz, W. J.; Herbinet, O.; Curran, H. J.; Silke, E. J. Combust. Flame 2009, 156 (1), 181. doi: 10.1016/j. combustflame.2008.07.014

(13) You, X.; Egolfopoulos, F. N.; Wang, H. Proc. Combust. Inst. 2009, 32 (1), 403. doi: 10.1016/j.proci.2008.06.041

(14) Zhou, P.; Hollis, O. L.; Crynes, B. L. Ind. Eng. Chem. Res. 1987, 26 (4), 846. doi: 10.1021/ie00064a038

(15) Herbinet, O.; Marquaire, P. M.; Battin-Leclerc, F.; Fournet, R. J. Anal. Appl. Pyrol. 2007, 78 (2), 419. doi: 10.1016/j. jaap.2006.10.010

(16) Dahm, K. D.; Virk, P. S.; Bounaceur, R.; Battin-Leclerc, F.; Marquaire, P. M.; Fournet, R.; Daniau, E.; Bouchez, M. J. Anal. 
Appl. Pyrol. 2004, 71 (2), 865. doi: 10.1016/j.jaap.2003.11.005

(17) Malewicki, T.; Brezinsky, K. Proc. Combust. Inst. 2013, 34 (1), 361. doi: 10.1016/j.proci.2012.06.156

(18) Dagaut, P.; Cathonnet, M.; Boettner, J. C.; Gaillard, F. Combust. Flame 1988, 71 (3), 295. doi: 10.1016/0010-2180(88)90065-X

(19) Petrova, M. V.; Williams, F. A. Combust. Flame 2006, 144 (3), 526. doi: 10.1016/j.combustflame.2005.07.016

(20) Coffee, T. P. Combust. Flame 1984, 55 (2), 161. doi: 10.1016/ 0010-2180(84)90024-5

(21) Bikas, G.; Peters, N. Combust. Flame 2001, 126 (1-2), 1456.

(22) Honnet, S.; Seshadri, K.; Peters, N. Surrogate Fuel for Kerosene. http://www.itv.rwth-aachen.de/fileadmin/downloads/. (accessed Oct 27, 2011)

(23) Olchanski, E.; Burcat, A. Int. J. Chem. Kinet. 2006, 38 (12), 703.

(24) Ranzi, E.; Frassoldati, A.; Granata, S.; Faravelli, T. Ind. Eng. Chem. Res. 2004, 44 (14), 5170.

(25) Battin-Leclerc, F.; Fournet, R.; Glaude, P.; Judenherc, B.; Warth, V.; Côme, G.; Scacchi, G. Proc. Combust. Inst. 2000, 28 (2), 1597. doi: 10.1016/S0082-0784(00)80557-3

(26) Lindstedt, R.; Maurice, L. J. Prop. Power 2000, 16 (2), 187. doi: $10.2514 / 2.5582$
(27) Kossiakoff, A.; Rice, F. O. J. Am. Chem. Soc. 1943, 65 (4), 590. doi: $10.1021 / \mathrm{ja} 01244 \mathrm{a} 028$

(28) Chakir, A.; Bellimam, M.; Boettner, J. C.; Cathonnet, M. Int. J. Chem. Kinet. 1992, 24 (4), 385.

(29) Tamura, T. High-Temperature Reaction Rates for Five- and Six-Carbon Saturated Alkanes. Lawrence Livermore National Lab, Report, 1987.

(30) Yao, T.; Zhong, B. J. Acta Phys. -Chim. Sin. 2013, 29 (2), 237. [姚通, 钟北京. 物理化学学报, 2013, 29 (2), 237.] doi: 10.3866/PKU.WHXB201211271

(31) Held, T. J.; Marchese, A. J.; Dryer, F. L. Combust. Sci. Tech. 1997, 123 (6), 107. doi: 10.1080/00102209708935624

(32) Zhang, Y. P.; Zhong, B. J. J. Tsinghua Univ. (Sci. Tech.) 2008, 48 (5), 904 . [张云鹏, 钟北京. 清华大学学报: 自然科学版, 2008, 48 (5), 904.]

(33) Kee, R. J.; Rupley, F. M.; Miller, J. A.; et al. CHEMKIN Release 4.1; Reaction Design: San Diego, CA, 2006.

(34) Li, J.; Shao, J. X.; Liu, C. X.; Rao, H. B.; Li, Z. R.; Li, X. Y. Acta Chim. Sin. 2010, 68 (3), 239. [李 军, 邵菊香, 刘存喜, 饶含兵, 李泽荣, 李象远. 化学学报, 2010, 68 (3), 239.]

(35) Rabitz, H.; Kramer, M.; Dacol, D. Annu. Rev. Phys. Chem. 1983, 34 (1), 419. doi: 10.1146/annurev.pc.34.100183.002223 